\section{Hormontherapie-Resistenz überwinden}

\begin{abstract}
Etwa $30 \%$ der Frauen mit Östrogenrezeptor-positivem (ER+) metastasiertem Mammakarzinom nach der Menopause entwickeln eine Resistenz auf eine Therapie mit Aromataseinhibitoren (Al). Frühe klinische Studien wiesen darauf hin, dass der mTOR-Inhibitor Everolimus neben einer Antitumoraktivität die Sensitivität resistenter Tumore wieder verbessern kann.
\end{abstract}

W ahrscheinlich sind verschiedene Signaltransduktionswege an der Hormonresistenz beteiligt, so auch der Phosphatidylinositol-3-Kinase (PI3K)/ AKT/mTOR-Signalweg. Eine randomisierte Phase-II-Studie untersuchte Wirksamkeit und Sicherheit des oralen mTOR-Inhibitors Everolimus in Kombination mit Tamoxifen versus Tamoxifen alleine bei 111 postmenopausalen Frauen mit metastasiertem, ER+, HER2negativem Mammakarzinom mit AI-Resistenz. Die Patientinnen waren median 64 Jahre alt, $78 \%$ wiesen Knochen- und $43 \%$ viszerale Metastasen auf. Die vorangegangene AI-Therapie hatten $41 \%$ adjuvant erhalten, $67 \%$ als Erstlinientherapie der metastasierten Erkrankung.

Im Kombinationsarm erhielten die Patientinnen täglich $20 \mathrm{mg}$ Tamoxifen und $10 \mathrm{mg}$ Everolimus ( $\mathrm{n}=54)$, im Kontrollarm nur Tamoxifen $(n=57)$. Als primären Endpunkt der Studie definierten die Autoren die Rate des klinischen Benefits (CBR), d. h. den prozentualen Anteil von Patientinnen, der nach sechs Monaten unter der Therapie mindestens eine stabile Erkrankung aufwies. Im Kombinationsarm lag die CBR nach sechs Monaten bei $61 \%$ (95\%-Konfidenzintervall [95\%-KI] 47-74\%), im Vergleichsarm bei $42 \%$ (95\%-KI 29-56\%). Die Zeit bis zur Progression (TTP) war bei Therapie mit Tamoxifen und Everolimus median um 4,1 Monate länger als bei Behandlung mit Tamoxifen alleine (8,6 vs. 4,5 Monate). Dies entspricht einer Risikoreduktion für eine Progression um 46\% (Hazard Ratio [HR] 0,54; 95\%-KI 0,36-0,81). Das Sterberisiko ver- ringerte sich durch die Kombination um $55 \%$ (HR 0,45; 95\%-KI 0,24-0,81).

Die Kombination ging allerdings auch mit mehr Nebenwirkungen einher, z. B. mit mehr Fatigue (72 vs. $53 \%$ ), Stomatitis (56 vs. $7 \%$ ), Hautausschlägen (44 vs. $7 \%$ ), Anorexie (43 vs. $18 \%$ ) und Diarrhö (39 vs. $11 \%$ ). Bei $20 \%$ der Patientinnen im Kombinationsarm führten die $\mathrm{Ne}$ benwirkungen zu einer Dosisreduktion, drei beendeten deswegen die Therapie. Die Häufigkeit schwerer Nebenwirkungen geben die Autoren aber für beide Gruppen gleichermaßen mit $32 \%$ an.

Fazit: Die randomisierte Phase-II-Studie weist darauf hin, dass die Therapie mit Everolimus zusätzlich zu Tamoxifen bei Vorliegen einer AI-Resistenz bei Frauen mit Mammakarzinom in der Postmenopause CBR, TTP und Gesamtüberleben verbessern kann.

Friederike Klein

Bachelot T et al. Randomized phase II trial of everolimus in combination with tamoxifen in patients with hormone receptor-positive, human epidermal growth factor receptor 2-negative metastatic breast cancer with prior exposure to aromatase inhibitors: A GINECO study. J Clin Oncol. 2012;30(22):2718-24.

\title{
Unter die Haut statt in die Vene
}

\section{Bisher erfolgte die Gabe von Trastuzumab zur Therapie des HER2-positiven Mammakarzinoms intravenös. In einer Phase-III-Studie wurde jetzt eine neue Galenik für die Subkutangabe geprüft.}

$\mathrm{D}$ ie offene Studie mit Patientinnen mit frühem, operablem HER2-positivem Mammakarzinom untersuchte, ob die subkutane (s.c.) Applikation pharmakokinetisch und bezüglich Wirksamkeit und Sicherheit der intravenösen (i.v.) nicht unterlegen ist. Randomisiert erhielten Patientinnen zusätzlich zu einer neoadjuvanten Chemotherapie in acht dreiwöchigen Zyklen alle drei Wochen Trastuzumab entweder i.v. mit einer Initialdosis von $8 \mathrm{mg} / \mathrm{kg}$ und einer Erhaltungsdosis von $6 \mathrm{mg} / \mathrm{kg}$ Körpergewicht $(n=299)$ oder s.c. in einer fixen Dosis von $600 \mathrm{mg}(\mathrm{n}=297)$. Die neoadjuvante Chemotherapie bestand aus vier Zyklen Docetaxel, gefolgt von vier Zyklen Fluorouracil, Epirubicin und Cyclophospha- mid. Nach der Operation wurde Trastuzumab ein Jahr lang weiter gegeben.

Während des achten Zyklus vor der Operation lagen die Talspiegel in der Gruppe mit s.c.-Gabe $33 \%$ über denen bei i.v.-Applikation. Bei 107 der 263 Patientinnen unter i.v.-Gabe (40,7\%) zeigte sich ein pathologisch komplettes Ansprechen (pCR), unter s.c.-Gabe bei 118 von 260 (45,4\%; Unterschied 4,7\%; 95\%KI 4,0-13,4\%). Damit war die s.c.- der i.v.-Applikation in beiden Endpunkten Talspiegel und pCR - nicht unterlegen.

Die häufigsten Nebenwirkungen waren Neutropenien (33,2 bei i.v.- vs. 29,0\% bei s.c.-Gabe), Leukopenien (5,7 vs. $4,0 \%$ ) und febrile Neutropenien (3,4 vs. $5,7 \%)$. Nebenwirkungen des Grads 3-4 waren bei s.c.-Gabe etwas häufiger (21 vs. $12 \%)$, v.a. schwere Infektionen und Infestationen ( 8,1 vs. $4,4 \%)$. Vier solcher Ereignisse führten in der neoadjuvanten Therapiephase zum Tode - eines in der i.v.-, drei in der s.c.-Gruppe; zwei unter s.c.-Trastuzumab-Therapie könnten mit der Behandlung zusammenhängen.

Fazit: Nach dieser Studie ist Trastuzumab, über fünf Minuten s.c. appliziert, der Infusion bezüglich Pharmakokinetik und Wirksamkeit nicht unterlegen. Auch das Sicherheitsprofil scheint vergleichbar, sodass die s.c.-Applikation mit Fixdosis (600 mg alle drei Wochen) nach der vom Hersteller initiierten Zulassungsstudie als Alternative zur Infusion infrage kommt.

Friederike Klein

Ismael G et al. Subcutaneous versus intravenous administration of (neo)adjuvant trastuzumab in patients with HER2-positive, clinical stage I-III breast cancer (HannaH study): A phase 3, openlabel, multicentre, randomised trial. Lancet Oncol. 2012;13(9):869-78. 